\title{
A nurse led programme improved physical function in older adults with chronic disease
}

\author{
Leveille SG, Wagner EH, Davis C, et al. Preventing disability and managing chronic illness in frail older adults: a randomized trial \\ of a community-based partnership with primary care.J Am Geriatr Soc 1998 Oct;46:1191-8.
}

\section{Question}

Can a multicomponent, senior centre (day hospital) programme led by a geriatric nurse practitioner (GNP) reduce disability, increase activity, and improve management of chronic disease in older adults?

\section{Design}

Randomised controlled trial (RCT) with 1 year of follow up.

\section{Setting}

A senior centre associated with 2 health maintenance organisations in northwest USA.

\section{Patients}

891 older adults were screened and 201 (mean age 77 y, 56\% women) were included. Inclusion criteria were age $\geqslant 70$ years, treatment for $\geqslant 1$ chronic condition excluding dementia or terminal illness, ability to walk independently and perform activities of daily living, non-participation in the senior centre programmes, and no cognitive impairment.

\section{Intervention}

Adults allocated to the intervention group $(n=101)$ met individually with the GNP to develop a targeted healthcare plan that addressed risk factors for disability and self management of their illness. The GNPs met with participants at the centre and made follow up telephone calls; volunteer mentors provided peer support. Physical activity and self management of disease were emphasised. 14 hours of group educational classes were given and adults were encouraged to take part in specific centre programmes. Adults allocated to the control group $(n=100)$ were given a tour of the centre, a schedule of events, and access to all centre activities.

\section{Main outcome measures}

Self reported physical function and disability (6 scales), physical performance ( 5 measures), healthcare utilisation, health behaviours (5 scales), and psychoactive drug use.

\section{Main results}

The median number of visits with the GNP was 3 per participant. Annual costs of the intervention were US\$300/ person. The GNP group showed improvement in activities of daily living $(p=0.014)$ and the number of disability days $(p=0.019)$. The groups did not differ for any of the performance measures. The GNP group had fewer total hospital days than the control group (33 v $116 \mathrm{~d}, \mathrm{p}=0.049)$ and showed a trend toward fewer hospital admissions (13 $v 22, \mathrm{p}=0.083)$. The GNP group had better scores on 2 measures of health behaviour related to exercise and physical activity $(\mathrm{p}=0.028$ and $\mathrm{p}=0.031$, respectively). The GNP group had higher rates of centre participation $(44 \% v 19 \%, \mathrm{p}<0.001)$ and lower rates of psychoactive drug use $(36 \%$ ข $20 \%, \mathrm{p}=0.039)$.

\section{Conclusion}

A multicomponent, senior centre programme led by a geriatric nurse practitioner for older adults with chronic disease increased physical activity and decreased difficulty with activities of daily living, total number of hospital and disability days, and use of psychoactive drugs.

Sources of funding: Retirement Research Foundation, Chicago, IL; Group Health Foundation; and SAFECO, Seattle, WA.

For correspondence: Dr S Leveille, Epidemiology, Demography, and Biometry Program, National Institute on Aging, 7201 Wisconsin Ave, Suite 3C-309, Bethesda, MD 20892, USA.Fax +1 301496 4006.

A modified version of this abstract appears in Evidence-Based Medicine.
The study by the multidisciplinary team of Leveille et al represents the first RCT to incorporate self management of chronic illness with disability prevention in frail older adults living in the community. Research related to self management of several chronic illnesses has shown reduced healthcare costs and burden of illness. ${ }^{1}$ RCTs on disability prevention in day hospitals, the medicalised equivalent to the study intervention, have contradictory results. Pitkala recently concluded that day hospital care does not reduce the use of other health services, nor improve physical function in older adults, but it does increase quality of life. ${ }^{2}$

The study is strong because it is community based, it reached older people previously not involved with the senior centre, the individualised health management goals were collaboratively planned with the GNP and physician, and preventive health and self management of chronic illness were emphasised. Sizeable cost benefits to healthcare insurers resulted from the study intervention.

Defined as frail, the study population was selected from a white, presumably English speaking, suburban area, but was still a high functioning group with $>84 \%$ having a high school education. This limits applicability to adults with variable cognitive problems, especially for disease self management. At baseline, more of the intervention participants were women, were not married, lived alone, had diabetes, and were more likely to report restricted activity days. The analysis employed many statistical measures with only some attaining significance. The mixed results of this study suggest the need for further investigation with a larger sample.
Community and institution based disability prevention programmes are dominated by case management models. ${ }^{3} \mathrm{~A}$ shift to self management of chronic illness with appropriate populations is a potentially cost effective, positive consideration for geriatric nurses. A GNP working collaboratively in a community setting can provide comprehensive health care including health promotion, which may help to prevent the cascade to disability.

Catherine Ford Thomas, RN, FNP, MS Instructor, School of Nursing University of Western Ontario London, Ontario, Canada

\footnotetext{
1 Landefeld CS, Chren MM. J Am Geriatr Soc 1998;46:1314-6.

2 Pitkala K. JAm Geriatr Soc 1998;46:1086-90.

3 Bernabei R, Landi F, Gambassi G, et al. BMJ
} 1998;316:1348-51. 